\title{
L'organisation du travail entre les responsables de cours et les tuteurs : un défi?
}

\section{Résumé}

Cette recherche tente de répondre à la question suivante: Quelle forme prend l'organisation du travail entre les responsables des cours et les tuteurs et quels en sont les impacts sur le travail des tuteurs? Des entrevues individuelles semi-dirigées auprès de 44 répondants provenant de 4 établissements d'enseignement, 3 canadiens et 1 européen, ainsi que 8 entrevues de groupe ont été réalisées. Les résultats montrent qu'il y a des difficultés importantes dans la gestion des tuteurs, qui est surtout déléguée aux responsables de cours (conseillers pédagogiques ou enseignants). Cette décentralisation ne favorise toutefois pas la prise de décision en équipe entre ces responsables, puisque nombre d'entre eux interviennent auprès d'un même tuteur et que chaque tuteur intervient auprès de plusieurs responsables. Ce manque de coordination entre les responsables rend invisible à l'établissement l'ensemble du travail de chaque tuteur, ce qui est accentué par le travail à domicile et à temps partiel. Les tuteurs ont ainsi la possibilité de prendre des décisions concernant les caractéristiques des enseignements, avec ou sans l'assentiment des responsables de cours, sans que personne en soit alerté.
L'organisation du travail entre les responsables et les tuteurs amène dans la majorité des cas ces derniers à travailler indépendamment les uns des autres plutôt qu'en équipe, chacun cherchant sa place dans la bonification des enseignements. Le manque de reconnaissance du travail des tuteurs a souvent pour effet de leur faire perdre toute motivation à prendre des initiatives, même lorsque les circonstances l'exigeraient.

\section{Mots-clés}

Organisation du travail, formation à distance, tuteurs, gestion décentralisée

\begin{abstract}
This research attempts to answer the following question: What is the form of the organization of work between the design teams and tutors and how does it impact the work of tutors? Semi-structured individual interviews with 44 respondents from four schools, three Canadian and one European, along with 8 group interviews were conducted. The results show difficulties that are particularly significant concerning the division of labour, the com-
\end{abstract}


munications between the stakeholders and the work management of tutors.

\section{Keywords}

Organization of work, distance education, tutors, division of labour

\section{Introduction}

La formation à distance (FAD) connaît de plus en plus d'adeptes, au détriment de la formation en présentiel (Dilworth et al., 2012; Karsenti, 2013). Toutefois, l'organisation du travail entre les responsables des cours et les tuteurs (aussi appelé encadrants, personnes tutrices ou correcteurs) en FAD semble rencontrer des problèmes importants, tant dans les établissements exclusivement destinés à la FAD que dans ceux qui offrent des formations bimodales. L'organisation du travail, selon Ketchum et Trist (1992, p. 178), « consiste à agencer les activités et les conditions dans lesquelles il doit se faire en fonction des caractéristiques qui lui donnent un sens ». Les employés désirent pouvoir exercer leurs capacités, exprimer leurs préférences et s'épanouir dans leur travail, ce à quoi répondrait le management orienté vers la participation qui se préoccupe de la personne dans sa totalité (Kocyba et Renault, 2007). Les buts poursuivis par rapport au travail des employés devraient être, selon Robbins, Coulter, Leach et Kilfoil (2012/2015), d'encourager l'équité et l'esprit d'équipe et de reconnaître le travail des employés à leur juste valeur, tout en s'assurant de la satisfaction de l'employeur quant au rendement des employés.

Cet équilibre en matière de satisfaction pour les employés comme pour les employeurs peut-il être atteint dans les conditions de travail qu'on retrouve en FAD? Decamps et Depover (2011) expliquent que les tuteurs travaillent normalement à distance physiquement les uns des autres ainsi que des responsables de cours, induisant un sentiment d'isolement. De la confusion et des tensions existent relativement au rôle des tuteurs et des équipes de conception (Bertin et Narcy-Combes, 2012; Racette, Poellhuber et
Bourdages-Sylvain, 2017). La coopération entre ces deux groupes d'acteurs est difficile à obtenir (Comas-Quinn, de los Arcos et Mardomingo, 2012).

Dans le cadre d'une recherche financée par le Conseil de recherches en sciences humaines (CRSH), la question que nous abordons est la suivante: Quelle forme prend l'organisation du travail entre les responsables des cours et les tuteurs, dans quatre établissements de FAD, et quels en sont les impacts sur le travail des tuteurs? Malgré l'abondance des travaux portant sur la FAD, peu de recherches se sont intéressées aux impacts que prennent les formes d'organisation par rapport aux intervenants, et encore moins par rapport au travail des tuteurs. Nous abordons la question des difficultés particulières associées à l'offre de cours à distance pour ces acteurs de la formation ainsi que certaines notions de centralisation et de décentralisation de la gestion. Nous présentons ensuite la méthodologie employée, les résultats obtenus et une discussion sur ces résultats. Nous concluons en suggérant quelques pistes de recherche qui permettraient d'éclairer davantage cette organisation du travail en FAD.

\section{L'offre de cours à distance}

La liberté pédagogique traditionnellement accordée aux enseignants dans les cours en présentiel est entravée en FAD par l'industrialisation de la formation qu'elle implique (Guillemet, 2004; Marchand, Loisier, Bernatchez et Page-Lamarche, 2002). Selon Guillemet (2004, p. 99), l'autonomie des professeurs « rend très problématique un fonctionnement basé sur la mise sur pied d'équipes multiqualifiées appelées à accomplir un grand nombre de tâches [...] mais aussi celui de l'utilisation croissante de personnel contractuel, qui apparaît désavantageux en raison de la perte de sens de la communauté académique susceptible d'en résulter ». Comme présenté au tableau 1, en FAD, l'enseignant n'est pas seul à intervenir dans la forme que prendra son cours (la conception), dans la façon de le rendre accessible aux étudiants (la diffusion), dans l'encadrement que les étudiants reçoivent (le tutorat) et dans le suivi des cours (la gestion des tuteurs et des 
cours offerts). L'enseignant en présentiel assume souvent ces tâches seul, ou avec une équipe beaucoup plus réduite, ce qui limite les possibles écarts entre les cours qui ont été planifiés et ceux que les étudiants reçoivent.

\section{Tableau 1}

Intervenants pouvant être impliqués dans un cours à distance

\begin{tabular}{|l|l|}
\hline & Cours à distance - Intervenants \\
\hline $\begin{array}{l}\text { Conception d'un } \\
\text { cours }\end{array}$ & $\begin{array}{l}\text { Enseignant ou concepteur } \\
\text { Conseiller pédagogique } \\
\text { Spécialiste en communication écrite } \\
\text { Spécialiste de l'audiovisuel } \\
\text { Graphiste } \\
\text { Technicien informatique } \\
\text { Spécialiste de l'édition }\end{array}$ \\
\hline Diffusion du cours & $\begin{array}{l}\text { Service de diffusion du matériel } \\
\text { Service de gestion des tuteurs } \\
\text { Service de gestion des séries } \\
\text { d'examens, } \\
\text { des surveillants et des lieux d'examens } \\
\text { Service informatique }\end{array}$ \\
\hline Encadrement des & $\begin{array}{l}\text { Tuteurs } \\
\text { Responsable du cours } \\
\text { Coordonnateur }\end{array}$ \\
\hline Suivi du cours & $\begin{array}{l}\text { Tuteur } \\
\text { Responsable du cours } \\
\text { Coordonnateur } \\
\text { Service de gestion des tuteurs }\end{array}$ \\
\hline
\end{tabular}

Parmi tout le personnel affecté au bon fonctionnement d'un cours, l'équipe de conception et les tuteurs constituent les acteurs clés, l'équipe de conception définissant ce que l'étudiant recevra et les tuteurs assurant l'encadrement des étudiants, d'où l'importance d'une délégation réussie aux tuteurs, phénomène propre à la FAD. Cette délégation est difficile du fait que l'équipe de conception n'existe que pour la période de conception du cours, le responsable désigné pour le cours en assurant ensuite la bonne marche. De plus, la tâche des tuteurs est mal définie et les tuteurs ne sont pas toujours réputés être experts dans les contenus des cours dont ils ont la charge (Loisier, 2013). Il est conséquemment difficile de faire une description précise de leur tâche : " Le travail du tuteur à distance recouvre néanmoins des fonctions larges, peu définies et en perpétuel renouvellement. » (Dir et Simonian, 2015, p. 2)

D'un autre côté, selon Wengrowicz et Offir (2013), le rôle des enseignants devrait être redéfini également pour la FAD. Dans les cours à distance, il faut exercer une gestion minutieuse du travail de conception des cours, laquelle implique, contrairement aux cours offerts en présentiel, l'intervention d'une équipe de spécialistes. Ces cours seront normalement d'une durée supérieure à quelques mois, comprendront des activités qui favorisent un apprentissage autonome, nécessitant des éléments de motivation particuliers et, finalement, s'adresseront à un bassin d'étudiants beaucoup plus important. Après avoir conçu les enseignements, l'enseignant (ou le concepteur) qui ne peut assumer lui-même l'encadrement des étudiants parce qu'ils sont en trop grand nombre le délègue à une tierce personne, qu'on appelle le tuteur, ce qui ne correspond nullement à la délégation de l'enseignement à un chargé de cours, puisque ce dernier donne le cours en plus d'encadrer les étudiants. Les tuteurs jouent un rôle intermédiaire entre l'enseignant, ou toute autre personne désignée comme étant responsable du cours, et les étudiants. Le responsable du cours doit ensuite faire le suivi des cours et des tuteurs pour assurer une diffusion harmonieuse des cours.

Relativement à ces tuteurs, comme l'exprime Loisier $(2013$, p. 26), « ces nouveaux intervenants [les tuteurs] bousculent les territoires de compétences et plus généralement la culture organisationnelle ». Gélis (2015) explique que cette séparation des tâches entre les membres des équipes de conception et des tuteurs devrait amener à une spécialisation en qualification et en professionnalisation ou à un appauvrissement des tâches et à leur déqualification, selon la gestion qui en est faite. Pelletier (2014) explique qu'il s'agit plutôt d'un appauvrissement des tâches, le travail se faisant surtout en silo. Mais, est-ce vraiment le cas? Si oui, pourquoi en est-il ainsi? Selon Depover et Quintin (2011), le matériel pédagogique est reconnu comme étant de haute 
qualité dans l'approche industrielle de la FAD, basée principalement sur des documents papier. Mais, par rapport aux cours offerts sur le Web, basés sur le modèle de l'exploitation des médias, il est selon eux plus difficile de coordonner plusieurs experts entre eux, dont les tuteurs qui doivent y jouer un rôle beaucoup plus significatif. Pour arriver à optimiser le travail de chacun, quelle forme de gestion peut-on appliquer aux équipes de conception et aux tuteurs en FAD? L'autonomie des enseignants devrait-elle être préservée dans cette gestion large des enseignements? Autrement dit, quel type de personnel décide (ou devrait décider) en matière de gestion des cours et des tuteurs et quels sont les éléments de gestion qui portent ombrage à une optimisation du travail?

\section{La centralisation par opposition à la décentralisation de la gestion}

La centralisation, inspirée des pratiques tayloristes, implique une prise de décision centralisée au niveau des hauts dirigeants de l'organisation. Lorsque le partage des tâches est standardisé et très détaillé et que la rémunération est à la pièce, c'est-à-dire associée à chaque tâche accomplie, il s'agit d'une gestion centralisée où la division du travail est appliquée à l'extrême. Cette pratique caractérisée par une standardisation de chacune des étapes du travail est dévalorisante pour les employés qui sont alors contraints à des formes de travail répétitives et peu enrichies qui les amènent à démissionner intérieurement. Selon Kocyba et Renault (2007), la vie véritable des employés dans un tel régime se déroule à l'extérieur du milieu de travail, le travail ne servant qu'à combler les besoins financiers. La division du travail dans une telle approche, où l'opinion de l'employé est ignorée à tous les niveaux, induit une insatisfaction professionnelle puisque les employés n'arrivent pas à s'accomplir (Robbins et al., 2012/2015, p. 185). Ces pratiques déshumanisent le travail en plus de limiter le transfert, voire d'entraîner la perte d'information.
La décentralisation de la gestion, contrairement à la centralisation, stimule l'autonomie tant désirée par les employés (Boisvert et al., 2011; Garrison, Chesley, Carroll et Webb, 2011). Elle permet d'alléger la direction qui, ayant peu de problèmes à résoudre, se concentre sur la stratégie d'ensemble. La décentralisation fournit aux gestionnaires subalternes une expérience en matière de prise de décision, ce qui favorise la satisfaction professionnelle. Toutefois, certains inconvénients sont attribués à la décentralisation, tels que les mauvaises décisions prises par les décideurs subalternes dues à un manque de coordination entre les preneurs de décision - chacun pouvant viser des objectifs différents de ceux de l'organisation dans son ensemble - ou, encore, une mauvaise évaluation des répercussions de chacune des décisions pour l'organisation. La qualité de la gestion y est donc variable, au gré du style de gestion de chaque décideur, compliquant également l'implantation de nouvelles pratiques.

Dans une certaine forme de décentralisation, où la prise de décision se fait en équipe, il peut être difficile d'arriver à décider, surtout lorsqu'il n'est pas possible pour un supérieur hiérarchique de trancher (Kocyba et Renault, 2007). La gestion en équipe peut irriter les employés, en plus d'induire, dans l'ensemble, une perte de visibilité et de reconnaissance pour le travail réalisé. Selon Kocyba et Renault (2007), lorsque le travail des employés est invisible aux niveaux supérieurs, les exigences formulées à l'endroit des employés peuvent être inappropriées puisque seuls les employés concernés savent exactement comment il convient de travailler. Dans ce contexte, lorsque personne n'est en mesure d'évaluer le travail réalisé, l'employé peut difficilement savoir où il en est. Bien que les employés aient besoin que leur travail soit reconnu, dans un tel contexte, " on peut constater, en même temps, une hostilité marquée contre certaines formes de "mise en évidence" des prestations individuelles, qui sont perçues comme surveillance, comme espionnage, comme signes de méfiance » (Kocyba et Renault, 2007, p. 114). 


\section{La méthodologie}

Cette recherche exploratoire est de type qualitatif (Creswell, 2012), ce qui offre une possibilité élevée de conduire à des questionnements de recherche plus en profondeur (Denzin, 2012). Elle ne vise pas l'exhaustivité, mais plutôt l'illustration. Les répondants, 22 tuteurs, 14 membres des équipes de conception et 8 directeurs administratifs, proviennent de 4 établissements : l'Université TÉLUQ, le Cégep à distance (CAD), la Faculté de l'éducation permanente de 1'Université de Montréal (FEP) et l'Éducation à distance de la Fédération WallonieBruxelles (EAD). Après avoir fait l'objet d'une validation interjuges, une grille d'entrevue a été distribuée à l'avance aux participants qui se sont portés volontaires pour participer à cette recherche. Des entretiens semi-dirigés ont été menés en présentiel ou sur Adobe Connect, puis analysés au regard d'une grille, non pas fondée sur des catégories prédéterminées, mais induite par une stratégie d'immersion et de cristallisation des données, afin de circonscrire les grandes tendances qui s'en dégagent. Une synthèse des réponses obtenues lors des rencontres individuelles a été présentée au personnel concerné de ces établissements, dans une vidéo accessible sur Moodle, afin de confirmer, d'infirmer ou de compléter ces résultats. Par rapport à chaque établissement, une nouvelle synthèse des résultats, enrichie des commentaires reçus, a servi de grille aux entrevues de groupe. Les propos tenus par les répondants ont été transcrits et codés avec un taux d'accord interjuges de plus de $80 \%$. Pour avoir une meilleure compréhension des résultats, les citations des tuteurs sont désignées par « $\mathrm{T} »$, celles des membres des équipes de conception par « $\mathrm{C} »$ et celles des directeurs administratifs par $« \mathrm{D} »$.

\section{Les résultats}

Nous décrivons tout d'abord les établissements qui ont participé à cette recherche. Nous présentons ensuite la situation des responsables des cours et celle des tuteurs dans l'organisation du travail qui les concerne ainsi que quelques solutions proposées par les répondants à certains problèmes soulevés.

\subsection{Les établissements participants}

Le tableau 2 présente les principales caractéristiques de chacun des établissements étudiés, soit leur date de création, le nombre de formations offertes à distance, le mode de diffusion des cours à distance et le rythme des inscriptions.

\section{Tableau 2}

Caractéristiques des établissements participants

\begin{tabular}{|l|l|l|l|l|}
\hline & \multicolumn{1}{|c|}{ EAD } & \multicolumn{1}{|c|}{ FEP } & \multicolumn{1}{c|}{ CAD } & \multicolumn{1}{|c|}{ TÉLUQ } \\
\hline $\begin{array}{l}\text { Date de } \\
\text { création }\end{array}$ & 1959 & $\begin{array}{l}1952 \\
\text { (date } \\
\text { inconnue } \\
\text { pour la } \\
\text { FAD) }\end{array}$ & 1991 & 1972 \\
\hline $\begin{array}{l}\text { Nombre de } \\
\text { formations } \\
\text { offertes à } \\
\text { distance }\end{array}$ & $\begin{array}{l}1000 \\
\text { modules }\end{array}$ & 26 cours & 250 cours & 400 cours \\
\hline $\begin{array}{l}\text { Mode de } \\
\text { diffusion } \\
\text { des cours à } \\
\text { distance }\end{array}$ & FAD & $\begin{array}{l}\text { Présentiel } \\
\text { et FAD }\end{array}$ & FAD & FAD \\
\hline $\begin{array}{l}\text { Rythme } \\
\text { d'inscription }\end{array}$ & En continu & $\begin{array}{l}\text { Par } \\
\text { cohorte }\end{array}$ & $\begin{array}{l}\text { En } \\
\text { continu }\end{array}$ & $\begin{array}{l}\text { En } \\
\text { continu }\end{array}$ \\
\hline
\end{tabular}

Trois des établissements étudiés offrent un grand nombre de cours à distance depuis plusieurs années, contrairement à la FEP, où la majorité des cours sont plutôt offerts en présentiel. Ce dernier établissement est également le seul à offrir ses cours en mode cohorte plutôt qu'en continu. Parmi les thématiques dominantes du discours des répondants, l'inscription en continu a été largement commentée par les participants, qui considèrent ce rythme d'inscription nuisible à plusieurs égards. Le suivi dans la remise des travaux des étudiants est beaucoup plus difficile. La prise de vacances des tuteurs implique qu'au cours de cette période, les étudiants ne bénéficient d'aucun encadrement ou, encore, que ces étudiants sont transférés à d'autres tuteurs. Les travaux et les examens ne peuvent pas 
tous être corrigés en même temps, ce qui alourdit la tâche des tuteurs et peut entraîner des incohérences dans la correction. En plus de ces problèmes liés à l'encadrement, l'inscription en continu empêche un enseignant ou un tuteur d'intervenir auprès de tous les étudiants pendant une même semaine de cours, puisque chaque étudiant suit son propre calendrier. Des modifications dans les cours ne peuvent être faites que pour une nouvelle version du cours, soit pour les étudiants à venir, sans quoi l'étudiant qui navigue dans le cours pourrait s'y perdre. Bien que l'inscription en continu présente beaucoup d'inconvénients, elle est malgré tout très appréciée par les étudiants, puisqu'elle leur permet de commencer et de terminer leurs cours au moment qui leur convient.

\subsection{La situation des responsables des cours}

Le tableau 3 présente le personnel responsable de la conception, du suivi des cours et des tuteurs ainsi que de la rédaction des grilles d'évaluation des établissements concernés.

\section{Tableau 3}

Les responsables, par établissement

\begin{tabular}{|l|l|l|}
\hline & ED - FEP - CAD & TÉLUQ \\
\hline $\begin{array}{l}\text { Responsable } \\
\text { de la } \\
\text { conception } \\
\text { des cours }\end{array}$ & $\begin{array}{l}\text { Conseillers } \\
\text { pédagogiques } \\
\text { (embauche de } \\
\text { concepteurs } \\
\text { contractuels) }\end{array}$ & Enseignants \\
\hline $\begin{array}{l}\text { Responsable } \\
\text { du suivi des } \\
\text { cours et des } \\
\text { tuteurs }\end{array}$ & $\begin{array}{l}\text { Conseillers } \\
\text { pédagogiques }\end{array}$ & Enseignants \\
\hline $\begin{array}{l}\text { Responsable } \\
\text { de la rédaction } \\
\text { des grilles } \\
\text { dévaluation }\end{array}$ & $\begin{array}{l}\text { Conseillers } \\
\text { pédagogiques } \\
\text { (le plus souvent) } \\
\text { ou concepteurs }\end{array}$ & Enseignants \\
\hline
\end{tabular}

Dans trois établissements à l'étude, ce sont les conseillers pédagogiques qui sont responsables de la conception des cours ainsi que du suivi des cours et des tuteurs. Les conseillers pédagogiques ont souvent à rédiger des grilles d'évaluation à la suite du travail réalisé par les concepteurs. Ces conseillers pédagogiques se sentent dépassés dans cette activité, n'étant pas experts dans ces disciplines. Il n'est donc pas étonnant que certains tuteurs critiquent ces grilles. «On les améliore à notre façon. Quand c'est bien bâti, c'est correct, mais dans mon cours, la grille n'évalue rien. Je me suis fait une nouvelle grille» $(\mathrm{T})$. Dans le quatrième établissement, ce sont des enseignants qui assument ces responsabilités. L'un des membres des équipes de conception explique : «L'enseignant est responsable de tout. Il délègue des tâches à des tuteurs, à des coordonnateurs, mais il garde la responsabilité $\mathrm{du}$ bon fonctionnement de ses cours » (C). Mais, selon les répondants, les enseignants auraient trop de cours à leur charge pour bien s'acquitter du suivi des cours et des tuteurs. Selon les responsables de cours, la tâche d'enseignement est très importante : « Mettre les cours à jour n'est pas facile. Certains ont jusqu'à 10 cours à leur tâche » (C). Cette organisation du travail amène certains problèmes aux responsables de cours.

Dans certains cas, les responsables de cours se plaignent de ne pas recevoir suffisamment d'information de la part des tuteurs sur le déroulement de leurs cours. Voici ce que certains répondants ont exprimé à ce sujet :

Je me suis rendu compte que certains tuteurs adaptaient les corrigés et gardaient ces modifications pour eux. (P)

Parfois le tuteur informe le professeur des problèmes, mais parfois, il ne le fait pas. (D)

Pour être mieux informés sur le déroulement de leurs cours, certains responsables utilisent d'autres moyens que celui de questionner leurs tuteurs, comme l'exprime ici un tuteur : "Il y a même un des enseignants qui me disait qu'il prenait des étudiants à sa charge, justement pour avoir des feed- 
backs sur les cours » $(\mathrm{T})$. Toutefois, certains tuteurs croient qu'il y a des responsables qui ne désirent pas être informés sur leurs cours. Voici l'opinion d'un tuteur et celle d'un directeur :

Je pense que les responsables de cours s'attendent à ne pas entendre parler de moi [tuteur], justement, ni des étudiants. (T)

Le coordonnateur était obligé d'intervenir auprès du responsable, car le tuteur n'avait pas de réponse de sa part. (D)

Toutefois, certains tuteurs et responsables affirment avoir une bonne communication entre eux, comme expliqué ici :

Parce qu'il y avait beaucoup d'étudiants qui se plaignaient de la charge de travail, nous avons travaillé ensemble sur Skype. Le cours a ensuite été mis à l'essai. Je suis maintenant plus familière avec le cours. (T)

D'habitude, nous sommes sur la même longueur d'onde. (C)

\subsection{La situation des tuteurs}

Dans les quatre établissements à l'étude, les tuteurs travaillent à partir de leur domicile et sont donc éloignés physiquement des équipes de conception ainsi que des autres intervenants qui bénéficient d'une place de travail à l'établissement. Ils travaillent à temps partiel, sauf à la TÉLUQ, où il est possible, pour un certain nombre de tuteurs, de faire ce travail à temps plein.

Les tuteurs sont rémunérés à l'acte (par activité corrigée, par courriel envoyé, etc.), par étudiant encadré ou par cours, ce dernier cas s'appliquant seulement aux cours offerts en mode cohorte. Lorsqu'ils sont rémunérés à l'acte, les tuteurs se plaignent de ne pas être rémunérés pour certains aspects de leur travail tels que les échanges entre tuteurs, les formations personnelles qu'ils reçoivent ainsi que les imprévus auxquels ils font face, entre autres, ceux qui sont liés à l'informatique. «Moi, je m'énerve. On n'est déjà pas très bien payé. Si en plus, on perd notre temps avec des problèmes informatiques, ça n'a pas de sens » (T). Dans trois établissements, les répondants disent ne pas être suffisamment rémunérés pour le travail réalisé. De plus, dans tous les établissements étudiés, la charge des tuteurs dépend du maintien de l'offre des cours dans lesquels ils interviennent. Ces tuteurs ne sont donc jamais assurés de leur emploi d'une session à l'autre.

Selon les répondants, plusieurs tuteurs se limitent à faire un travail de correction. Les initiatives qui permettraient de bonifier les cours ne sont pas encouragées. Seuls les plus audacieux prennent en charge le bon fonctionnement des cours lorsque les équipes de conception sont sourdes à leurs demandes. Un des responsables de cours explique le rôle mal défini des tuteurs de la façon suivante: « Jusqu'où peut aller un tuteur dans la modification de certains éléments d'un cours, pour un tableau qui n'est plus à jour, par exemple? » (C). La liberté pour les tuteurs de faire plus, ou autrement, que ce qui a été planifié par l'équipe de conception dépend de l'attitude du responsable de chaque cours à l'égard d'une telle implication ou, encore, de l'invisibilité des actions des tuteurs aux yeux de ces responsables.

Bien que le suivi des étudiants, qui consiste à contacter les étudiants lorsque la date de remise des travaux est dépassée, fasse partie de la tâche des tuteurs, plusieurs d'entre eux affirment ne pas le faire. « Faire le suivi du cheminement des étudiants ne devrait pas nous revenir, puisque ce sont des adultes » $(\mathrm{T})$. Les tuteurs peinent à suivre le cheminement des étudiants, particulièrement dans un système d'inscription en continu. " Il est impossible d'avoir un calendrier pour chacun des 160 étudiants que j'encadre » $(T)$. De plus, les tuteurs critiquent le fait que les tâches administratives alourdissent beaucoup leur travail. Voici ce que certains tuteurs et directeurs ont exprimé à ce sujet : 
En plus de corriger, il faut faire imprimer les travaux, les mettre dans des enveloppes, les faire parvenir aux étudiants, entrer les notes des étudiants, faire le suivi de ceux qui n'envoient pas leurs travaux. (T)

Je n'aime pas faire des tâches de secrétaire, parce que je ne suis pas formée pour être secrétaire. (T)

Comme l'exprime ici un directeur, les tuteurs disent manquer d'aide pour faire un bon travail :

Les tuteurs se plaignent qu'on ne leur a pas dit comment corriger, comment tutorer. (D)

C'est important que des outils soient créés... Il faut que le tuteur ait quelque chose sur quoi se reposer. (D)

Pourtant, plusieurs tuteurs demandent de participer à l'amélioration des cours, d'être plus impliqués. En fait, c'est leur expérience d'intervenant auprès des étudiants qu'ils aimeraient mettre à profit lors de la conception des cours. Ils demandent de faire un travail à la hauteur de leurs qualifications plutôt que davantage de travail administratif.

Les tuteurs des quatre établissements considèrent leur rôle trop limité. Ils aimeraient contribuer à l'amélioration des cours. «Mes étudiants me parlent beaucoup, beaucoup. Ce que je peux apporter à l'équipe de conception, c'est une vision très réelle de la situation des étudiants » $(\mathrm{T})$. De plus, ils désirent avoir la possibilité d'assumer des mandats spéciaux, au-delà de leur tâche d'encadrement, ce qui est interdit dans le cas des tuteurs syndiqués. Mais, selon nos résultats, cette prise en charge de mandats spéciaux ne semble pas être une pratique courante dans les autres établissements non plus.

Les tuteurs manquent de reconnaissance et leur travail semble ignoré dans plusieurs cas, comme l'indique ce qui suit :

Les tuteurs ne sont pas suffisamment consultés et pas respectés dans leur rôle de tuteur. (C)
Chaque année, je me dis : Est-ce que je le fais encore? Pourquoi est-ce que je fais ça? (T)

La communication peut être faite par courriel, mais c'est généralement pour faire des reproches. (T)

En général, lorsque le responsable du cours ne corrige pas les problèmes soulevés, les tuteurs arrêtent de communiquer avec lui, tout simplement. (P)

Les tuteurs se disent souvent dépourvus face aux questions des étudiants parce qu'ils ne sont pas suffisamment formés par rapport aux cours et qu'ils ne reçoivent pas toujours les réponses aux questions qu'ils posent aux responsables de cours. De plus, plusieurs tuteurs ont une connaissance limitée de l'organigramme de l'établissement et du rôle de chacun.

Finalement, les tuteurs se plaignent de recevoir des responsables de cours des directives qu'ils jugent parfois irréalistes. Ils décident donc eux-mêmes des directives qu'ils suivront ou pas. Plusieurs commentaires vont en ce sens :

Les directives reçues: c'est un autre exemple du tricotage individuel que les tuteurs vont faire, selon leur manière de travailler. $(\mathrm{T})$

J'ai un peu de difficultés avec les directives. ( $\mathrm{T}$ )

Les directives ne sont pas suivies, ça non. (C)

J'attends des tuteurs qu'ils prennent le temps de lire le cours, qu'ils lisent les travaux, les guides de correction. Cela devrait être une obligation au point de départ. Certains lisent en diagonale, d'autres ne lisent pas, d'autres lisent au complet. On a toutes sortes de profils. (C) 
Comme il y a plusieurs tuteurs dans un même cours et que chaque tuteur travaille avec plusieurs responsables de cours, les façons de faire diffèrent beaucoup d'une situation à l'autre.

\section{Quelques solutions proposées par les répondants}

Une façon de rencontrer l'objectif des tuteurs, qui est d'intervenir dans la conception des cours et d'augmenter l'efficacité du travail, serait que chaque tuteur produise régulièrement un rapport (par session ou par année) et le remette aux responsables des cours, ou encore, qu'une réunion annuelle permette d'échanger sur les cours. Un des directeurs administratifs soutient cette idée : « Je pense que les réunions en face à face, avec les équipes, devraient avoir lieu au moins une fois par année » (D). Bien que ces recommandations puissent bonifier le travail d'enseignement dans l'ensemble, il semble que ce soit insuffisant. Un des établissements fait déjà de telles interventions et ses tuteurs considèrent néanmoins ne pas être utilisés à la mesure de leur talent.

Pour éviter que les courriels demeurent sans réponse, dans deux des quatre établissements à l'étude, la communication entre les équipes de conception et les tuteurs est centralisée dans un service de tutorat, dans un cas, et dans une adresse courriel, dans l'autre, et ce, afin de faire un meilleur suivi des échanges. Mais, bien que cette centralisation permette de régler les problèmes importants, elle limite les contacts, déjà jugés trop peu nombreux, entre les tuteurs et les responsables de cours. Certains pensent même que la communication ne serait pas plus efficace pour autant. « Autant la communication était éclatée, mais plus performante à l'époque, autant elle est plus centralisée aujourd'hui, mais n'est pas encore suffisamment forte et structurée » $(\mathrm{T})$. Les tuteurs demandent qu'il y ait davantage de rencontres en face à face afin de faciliter les entretiens virtuels par la suite. Mais, tout au moins, ils désirent que des rencontres en synchrone aient lieu afin d'avoir des échanges constructifs, comme le suggèrent Kocyba et Renault (2007, p. 16) : « Les communications verbales permettent de gagner en rapidité, en clarté et en harmonie. »

\section{Discussion}

Dans les quatre établissements à l'étude, la prise de décision concernant les cours et les tuteurs est décentralisée au niveau des responsables de cours, soit aux conseillers pédagogiques ou aux enseignants. Toutefois, les tuteurs n'ont pratiquement pas droit à la prise de décision. Nous pourrions croire que la relation directe entre les responsables-enseignants et les tuteurs est le gage d'une bonne coopération, mais les résultats présentés ne le confirment pas. Les responsables-enseignants disent manquer de temps pour faire une bonne gestion des cours et des tuteurs. Quant aux responsables-conseillers pédagogiques, ils ont de la difficulté à répondre aux questions de contenu disciplinaire. Mais dans tous ces cas, les responsables de cours travaillent peu ou pas du tout ensemble, c'est-à-dire en équipe, relativement au suivi des tuteurs. Étant donné le grand nombre de responsables de cours qui interviennent auprès de chaque tuteur, il n'y a pas de vue d'ensemble du travail de chacun. La figure 1 donne une idée de cette complexité dans la gestion des tuteurs. 


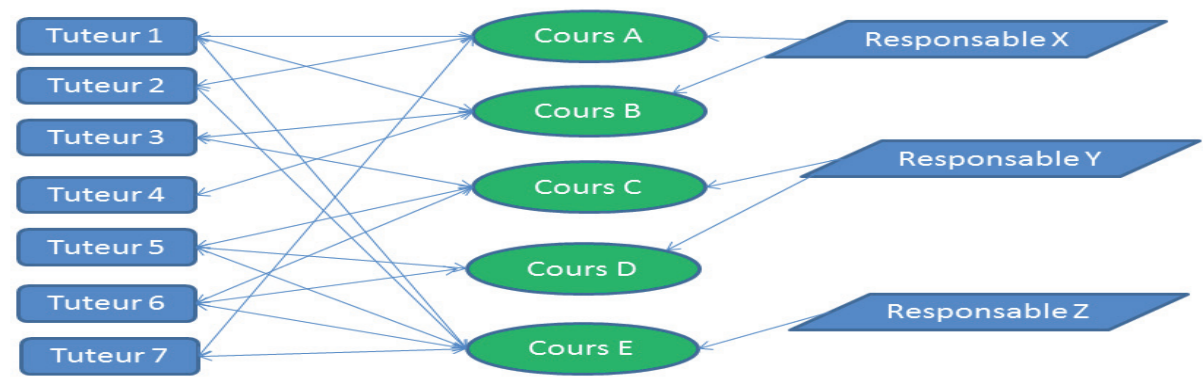

\section{Figure 1}

Liens entre tuteurs et responsables de cours en FAD

Dans cette figure, le tuteur 1 intervient dans les cours A, B et E, dont les cours A et B sont supervisés par $\mathrm{X}$ et le cours $\mathrm{C}$, par $\mathrm{Y}$. Le tuteur 2 intervient dans les cours A et E, dont le cours A est supervisé par $X$ et le cours $E$ par $Z$. Le superviseur $X$ doit donc faire le suivi des tuteurs 1,2 et 7 dans le cours A et des tuteurs 1, 3 et 4 dans le cours B.

Étant donné l'insatisfaction exprimée par les répondants sur cette organisation du travail, il semble que les responsables, qu'il s'agisse d'enseignants ou de conseillers pédagogiques, ne bénéficient pas des moyens nécessaires pour réaliser une gestion efficace des enseignements. Des règles uniformes devraient être adoptées, connues et appliquées, de façon à ce que les tuteurs y voient une cohérence et soient reconnus pour ce qu'ils font. En plus de se sentir laissés à eux-mêmes, les tuteurs ont une tâche très lourde, semble-t-il, puisqu'ils interviennent dans un grand nombre de cours et, pour trois établissements à l'étude, dans un mode d'inscription en continu.

Bien que la centralisation des communications, entre tuteurs et responsables de cours, exercée dans deux établissements permette d'assurer un meilleur suivi des demandes des uns et des autres, nous constatons que cette centralisation rencontre aussi ses limites. Elle implique une multiplication des intermédiaires et une perte de vue de l'identité de chacun. Toutefois, à la lumière des résultats obte- nus, et étant donné l'ampleur que prend le travail de tutorat en FAD, il semble qu'il s'agisse là d'un passage obligé, standardisant l'information transmise, les exigences formulées, l'évaluation du travail d'encadrement et la prise en compte des commentaires, plaintes ou éloges formulés sur les cours par les tuteurs.

Les rencontres en présentiel entre le responsable de cours et les tuteurs d'un même cours, demandées par les tuteurs, sont difficiles à réaliser dans un même temps, à cause des interrelations qui existent avec plusieurs équipes. Par exemple, le tuteur 1, de la figure 1 , ne peut pas se présenter en même temps à l'équipe du responsable $\mathrm{X}$ et du responsable $\mathrm{Z}$. D'un autre côté, ces rencontres deviennent très coûteuses lorsqu'elles sont organisées à différents moments, par équipe, étant donné que les heures de présence et les frais de déplacement sont assumés par les établissements impliqués, un tuteur devant avoir ainsi autant de rencontres que de cours dans lesquels il intervient.

Bien que les tuteurs détiennent une connaissance fine de la réaction des étudiants relativement à chaque cours, ils ont de la difficulté à parler de leur expérience aux responsables de cours, en face à face ou virtuellement, en mode synchrone, ce qui exerce une contrainte importante sur l'esprit d'équipe, autant pour les tuteurs envers les responsables de cours qu'entre les tuteurs pour un même cours. 
Le travail à domicile, qui repose surtout sur des communications par courriel et par téléphone avec les étudiants, traduit une certaine invisibilité du travail des tuteurs aux yeux des responsables. Il est alors difficile de valoriser et de reconnaître le travail des tuteurs pour ce qu'il est, comme le rapportent Kocyba et Renault (2007), sans compter que, dans ce contexte, les exigences formulées à leur endroit peuvent leur sembler irréalistes. Une certaine forme d'échanges devrait être instaurée entre les tuteurs et les responsables des cours, que ce soit par une gestion centralisée des communications, par la livraison de rapports annuels par les tuteurs, par des rencontres plus fréquentes ou par une présence accrue à l'établissement, en leur allouant des bureaux de travail, par exemple.

Relativement à la rémunération à l'acte, par étudiant encadré ou par cours, l'organisation du travail dans les quatre établissements étudiés se rapproche des pratiques inspirées du taylorisme où les initiatives ne sont pas valorisées. Les tuteurs n'ont ainsi aucun incitatif qui les encouragerait à effectuer des tâches pour lesquelles ils ne sont pas rémunérés, ce qui limite la possibilité de pallier les problèmes particuliers qui peuvent se présenter.

\section{Conclusion}

Quelle forme prend l'organisation du travail entre les responsables de cours et les tuteurs, dans quatre établissements de FAD, et quels en sont les impacts sur le travail des tuteurs? Nous constatons que l'organisation du travail dans les établissements étudiés est décentralisée, sans toutefois que des moyens adéquats permettent de l'appliquer avantageusement. Ainsi, autant les enseignants que les conseillers pédagogiques ont de la difficulté à bien s'acquitter de cette tâche. Toutefois, relativement au travail des tuteurs, la prise de décision est fortement centralisée au niveau des responsables de cours. Nous pouvons croire que cette délégation de gestion des enseignements aux responsables de cours tire son origine des pratiques exercées dans les cours en présentiel. Mais les différences importantes dans le travail qu'implique l'offre de cours à distance comparativement aux cours en présentiel exigent des procédés de gestion adaptés. Ketchum et Trist (1992) expriment bien ce fait en affirmant que les activités et les conditions de travail doivent refléter les caractéristiques de l'organisation qui leur donne un sens. Guillemet (2004) et Pelletier (2014) parlent de cette perte de sens dans le travail effectué dans les cours à distance.

À la lumière de ces résultats, il nous semble que les responsables devraient exercer leur gestion en équipe, bien que Kocyba et Renault (2007) voient dans cette forme de gestion un risque de perte de visibilité et de reconnaissance du travail des employés. Dans la présente situation, l'absence de coordination entre les responsables de cours augmente justement ce manque de visibilité et de reconnaissance. Toutefois, d'autres recherches pourraient explorer cette solution ou d'autres façons d'organiser ce travail d'enseignement en FAD.

Le travail à domicile et à temps partiel des tuteurs doit être remis en question afin d'impliquer davantage les tuteurs dans les équipes de conception et, par la même occasion, dans la prise de décision, et de permettre ainsi l'amélioration de la qualité des cours et de leur emploi. Quant à l'inscription en continu, il y a lieu de se demander si les avantages surpassent les coûts importants qu'elle implique puisque les tuteurs peinent à faire le suivi des étudiants comme le demandent les responsables. Nous faisons tout au moins le constat, comme l'explique Loisier (2013, p. 70), qu'en comparaison avec les cours en présentiel, «[d]évelopper des formations en FAD demande plus de ressources, de temps et d'organisation ».

Bien que certaines équipes semblent avoir adopté de bonnes pratiques, cette recherche met en évidence le fait que plusieurs responsables et tuteurs ont du mal à coopérer de façon à augmenter la valeur de leur travail respectif. Dans plusieurs cas, ces employés s'ignorent. En somme, l'organisation de ce travail d'enseignement dans les établissements étudiés a pour conséquence de séparer fortement les responsables et les tuteurs, ce qui en complique 
la gestion, bien que nous estimions que ce ne soit pas là un résultat visé par les dirigeants. Ainsi, il $\mathrm{y}$ a une distance physique (travail à domicile par rapport au travail à l'établissement) ainsi qu'une distance relative à la séquence du travail (l'encadrement succède à la conception), aux intervenants impliqués (travail avec les étudiants par rapport au travail avec l'équipe de conception) et au statut d'emploi (temps partiel par rapport au temps plein). La forme de rémunération offerte, qui consiste à payer à l'acte, par étudiant encadré, n'encourage nullement les initiatives et laisse peu de marge de manœuvre pour que les tuteurs traitent correctement les cas d'exception. Ce travail invisible les prive également d'une reconnaissance pour le travail bien fait. Les tuteurs n'ont pour seule valorisation que l'appréciation de leurs étudiants, qu'ils ne voient jamais et qui ne sont que de passage. Il n'est donc pas étonnant que certains tuteurs se sentent démotivés.

Nous constatons que cette organisation du travail est, tout comme la FAD, en constante évolution. Mais, étant donné la compétition importante que se livrent les établissements dans ce créneau de la formation, la qualité de l'organisation du travail peut être déterminante pour son avenir. Des mesures devraient être instaurées pour que les preneurs de décision puissent davantage se coordonner et mettre à profit l'expérience des tuteurs. Ces derniers demandent d'être mieux épaulés et mieux compris dans leur travail, souvent complexifié par le nombre élevé de cours dans lesquels ils interviennent, par le nombre de responsables avec lesquels ils doivent travailler ainsi que par les types d'intervention à réaliser (correction, explication des contenus disciplinaires et des procédures méthodologiques ou informatiques, encouragement, détection des erreurs dans les cours, etc.). Une meilleure coordination entre les responsables et les tuteurs devrait permettre que leurs tâches respectives soient plus riches de retombées, pour eux-mêmes et pour l'établissement, mais surtout pour les étudiants.

\section{Références}

Bertin, J. C. et Narcy-Combes, J. P. (2012). Tutoring at a distance: Modelling as a tool to control chaos. Computer Assisted Language Learning, 25(2), 111-127. doi:10.1080 /09588221.2011.639785

Boisvert, H., Brouillette, M. C., Caron, M. A., Jacques, R., Laurin, C. et Mersereau, A. (2011). La comptabilité de management, prise de décision et contrôle ( $5^{\mathrm{e}}$ éd.). Montréal, Canada : ERPI.

Comas-Quinn, A., de los Arcos, B. et Mardomingo, R. (2012). Virtual learning environments (VLEs) for distance language learning: Shifting tutor roles in a contested space for interaction. Computer Assisted Language Learning, 25(2), 129-143. doi:10.1080/09588221.2011.636055

Creswell, J. W. (2012). Educational research: Planning, conducting and evaluating quantitative and qualitative research. Boston, MA : Pearson.

Decamps, S. et Depover, C. (2011). La perception du tutorat par les acteurs de la formation à distance. Dans C. Depover, B. De Lièvre, D. Peraya, J. J. Quintin et A. Jaillet (dir.), Le tutorat en formation à distance (p. 109-124). Bruxelles, Belgique : De Boeck.

Denzin, N. K. (2012). Triangulation 2.0. Journal of Mixed Methods Research, 6(2), 80-88. doi : $10.1177 / 1558689812437186$

Depover, C. et Quintin, J.-J. (2011). Tutorat et modèle de formation à distance. Dans C. Depover, B. De Lièvre, D. Peraya, J.-J. Quintin et A. Jaillet (dir.), Le tutorat en formation à distance (p. 15-27). Bruxelles, Belgique : De Boeck.

Dilworth, P., Donaldson, A., George, M., Knezek, D., Searson, M., Starkweather, K., ... Robinson, S. (2012). Editorial: Preparing teachers for tomorrow's technologies. Contemporary Issues in Technology and Teacher Education, 12(1), 1-5. Récupéré de http://learntechlib.org

Dir, M. et Simonian, S. (2015). Tuteur en ligne : une activité de travail aux prises avec ses contradictions. Dans A. Jézégou, P.-A. Caron et J. Heutte (dir.), Actes du colloque e-Formation 2015 (p. 45-49). Récupéré du site du colloque : http://www.trigone.univ-lille1.fr/eformation2015

Garrison, R. H., Chesley, R., Carroll, R. F. et Webb, A. (2011). Fondements de la comptabilité de gestion ( $2^{\mathrm{e}}$ éd.) (H. Bergeron et C. Roy, adapt.). Montréal, Canada : Chenelière/McGraw-Hill.

Gélis, J. M. (2015). Des responsables d'équipe dans le département d'un enseignement à distance : entre innovation, industrialisation et modèles de dissémination. Distances et médiations des savoirs, 10. Récupéré de http://journals.openedition.org/dms 
Guillemet, P. (2004). L'industrialisation de la formation, la fin d'un paradigme? Distances et savoirs, (2), 93-118. Récupéré de http://cairn.info/revue-distances-et-savoirs.htm

Karsenti, T. (2013). Les MOOC : révolution ou simple effet de mode? Revue internationale des technologies en pédagogie universitaire, 10(2), 6-22.

https://doi.org/10.18162/ritpu.2013.227

Ketchum, L. D. et Trist, E. (1992). All teams are not created equal. How employee empowerment really works. Londres, R.-U. : Sage.

Kocyba, H. et Renault, D. (2007). Reconnaissance, subjectivisation, singularité. Travailler, 2(18), 103-118. https://doi.org/10.3917/trav.018.0103

Loisier, J. (2013). Mémoire sur les limites et défis de la formation à distance au Canada francophone. Document préparé pour le Réseau d'enseignement francophone à distance du Canada (REFAD). Récupéré de http://refad.ca

Marchand, L., Loisier, J., Bernatchez, P.-A. et Page-Lamarche, V. (2002). Guide des pratiques d'apprentissage en ligne auprès de la francophonie pancanadienne. Document préparé pour le Réseau d'enseignement francophone à distance du Canada (REFAD). Récupéré de http://refad.ca

Pelletier, P. (2014). L'apport des sciences de la gestion à la compréhension du phénomène de la formation à distance en ligne. Revue internationale des technologies en pédagogie universitaire, 11(2), 52-71. https://doi.org/10.18162/ritpu.2014.249

Racette, N., Poellhuber, B. et Bourdages-Sylvain, M.-P. (2017). Quelles sont les caractéristiques de l'emploi et du travail des tuteurs en formation ouverte et à distance? Distances et médiations des savoirs, 18. Récupéré de http://journals.openedition.org/dms

Robbins, S. P., Coulter, M., Leach, E. et Kilfoil, M. (2015). Management (L. Hamel, trad.). Montréal, Canada : ERPI. (Ouvrage original publié en 2012 sous le titre Management (10th ed.). Toronto, Canada : Pearson Education).

Wengrowicz, N. et Offir, B. (2013). Teachers' perceptions of transactional distance in different teaching environments. American Journal of Distance Education, 27(2), 111-121. doi:10.1080/08923647.2013.773701 